Bryn Mawr College

Scholarship, Research, and Creative Work at Bryn Mawr

College

1980

\title{
A deuterium nuclear magnetic resonance study of chain disorder in lamellar potassium palmitate: The effect of long and short chain guests
}

Peter A. Beckmann

Bryn Mawr College, pbeckman@brynmawr.edu

E. E. Burnell

M. A. Heldman

K. R. Northey

T. P. Higgs

Let us know how access to this document benefits you.

Follow this and additional works at: https://repository.brynmawr.edu/physics_pubs

Part of the Physics Commons

\section{Custom Citation}

Beckmann, Peter A., E. E. Burnell, M. A. Heldman, K. R. Northey, and T. P. Higgs. 1980. "A deuterium nuclear magnetic resonance study of chain disorder in lamellar potassium palmitate: The effect of long and short chain guests." Canadian Journal of Physics 58.11: 1544-1554.

This paper is posted at Scholarship, Research, and Creative Work at Bryn Mawr College. https://repository.brynmawr.edu/physics_pubs/115

For more information, please contact repository@brynmawr.edu. 


\title{
A deuterium nuclear magnetic resonance study of chain disorder in lamellar potassium palmitate: The effect of long and short chain guests
}

\author{
P. A. Beckmann, E. E. Burnell, M. A. Heldman, K. R. Northey, and T. P. Higgs \\ Canadian Journal of Physics 58.11: 1544-1554. https://doi.org/10.1139/p80-203
}

\begin{abstract}
The deuterium magnetic resonance spectra of lamellar phases of host perdeuterated potassium palmitate mixed with varying amounts of guest protiated potassium octanoate and behenate and $86.3 \mathrm{~mol} \% \mathrm{D}_{2} \mathrm{O}$ are reported. At higher temperatures, the short chain guests give rise to more fluidity and the long chain guests give rise to less fluidity towards the end of the host chain. At low temperatures there are changes in the interaction between the polar head group and the water which depend on sample composition. The transition from the lamellar phase to the low temperature gel phase is investigated and in some cases the gel phase is found to be a complicated many-phase region, at least for the water. The spectral results are presented in detail but compact and empirical methods of analysis are also investigated.
\end{abstract}

\section{Introduction}

Mixtures of fatty acid salts and water form a variety of lyotropic crystalline phases (1) which are of great interest in surface and interface science. The liquid crystalline or $\mathrm{L}_{\alpha}$ lamellar phase is of particular interest because the bilayer arrangement of the soap molecules is the same as that formed by the phospholipids of biological membranes. Such a model is greatly oversimplified since real biological membranes also contain proteins imbedded in the bilayer, and in addition the phospholipids have various chain lengths. This paper addresses the latter complication. We have studied the effect that added guest soap molecules of differing chain lengths have on the molecular order of a more abundant host soap molecule. In particular, we have investigated the fluidity of the lipid chains in perdeuterated potassium palmitate $\left(\mathrm{CD}_{3}\left(\mathrm{CD}_{2}\right)_{14}\right.$, $\mathrm{COOK}$ in the presence of varying amounts of protiated potassium octanoate $\mathrm{CH}_{3}\left(\mathrm{CH}_{2}\right)_{20} \mathrm{COOK}$. It has been found that for low mole fraction of guest molecules the mean area per polar head in the $\mathrm{L}_{\alpha}$ phase is not affected whereas when the mole fraction of guests exceeds about $20 \%$ the lamellar phase is no longer stable (2). In our experiments, concentrations of the guest molecule are sufficiently low that the lamellar nature of the system is not affected.

The experimental techniques is Fourier transform quadrupolar echo deuteron magnetic resonance spectroscopy (3). The non-zero electric field gradients at the deuterium nuclei lead to quadrupolar splittings of the nmr lines. These splittings are large compared with the broadening resulting from dipolar interactions and magnetic field inhomogeneities and depend on the degree to which the quadrupolar interactions are averaged out by the molecular motion. Thus one obtains a deuterium nmr spectrum with small splittings for the $\mathrm{CD}_{3}$ deuterons at the end of the chain where there may be consideration motion and large splittings for the $\alpha$ deuterons where there is considerably less motion (4).

Studies related to those presented here have appeared in the literature. Lamellar phases of mixtures of potassium decanoate, meristate, and stearate at $60^{\circ} \mathrm{C}$ have been studied (5) and it was found that there is little change in the methylene chain deuterium splittings upon addition of guest molecules. The deuterium splitting of the $a$ position in fatty acids and fatty acid salts dissolved in small concentrations in orienting lyotropic liquid crystals has been measured (6), 
and for short chain lengths a linear dependence of splitting on chain length was observed. These studies did not examine the temperature dependence of the splittings, as is done in the present study.

\section{Experimental}

Palmitic acid (Sigma Chemical Co., Sigma grade, 99\%) was used to prepare palmitic- $d_{31}$ acid as discussed elsewhere (7). The deuterated acid was purified by silica gel chromatography. The incorporation of deuterium was found to be greater than $98.5 \%$ by high resolution nmr and mass spectrometric analysis.

The potassium salts were precipitated from ethanol by the addition to the corresponding fatty acids of 1 equiv. of potassium hydroxide in concentrated aqueous solution. The potassium salts were then recrystallized from ethanol and vacuum dried at room temperature to yield the soap as a fluffy white solid.

Several samples of 6.3 mol $\mathrm{D}_{2} 0$ to 1 mol soap were prepared by weighing the soap and $\mathrm{D}_{2} 0$ to $1 \%$ accuracy into a constricted Pyrex sample tube. The soap was mainly potassium palmitate, but also contained $0,0.01,0.05$, or $0.20 \mathrm{~mol}$ of potassium octanoate or potassium behenate per mole total soap. For reference, Table 1 lists the composition of the various mixtures used, along with a code which will be used hereafter to identify the various samples. Samples were mixed by centrifuging many times through the constriction in the sealed glass tube used for nmr. It should be noted that even after 50 such mixings spectral changes were observed to occur on further mixing; hence, samples were mixed (typically >100 times) until no further spectral changes occurred.

Deuterium nmr spectra were obtained as a function of temperature in the lamellar and lower temperature phases at $13.8 \mathrm{MHz}$ with a Bruker SXP 4-100 pulsed nmr spectrometer by Fourier transforming the signal starting at the top of the quadrupolar echo (3). Spectra were run on resonance and Fourier transformation gave half of the symmetric spectrum. The spectra obtained for $0 \%$ guest differ slightly from those previously reported (8-10). These differences were traced via mass spectrometry to a greater amount of different chain length fatty acids (e.g., stearic acid) in the palmitic acid (supplied from Sigma Chemical Co.) which was used to prepare the perdeuterated potassium palmitate. For the present study we are interested only in changes which occur upon addition of different chain length fatty acid salts, and hence the minor differences compared with previous work need not concern us further. The present set of samples forms a useful basis for the comparisons of interest.

\section{Results and Discussions}

The deuterium nmr of the six samples indicated in Table 1 has been studied as a function of temperature in the $\mathrm{L}_{\alpha}$ and some lower temperature gel or $\mathrm{L}_{\beta}$ phases. First, we discuss the results for the lower temperature phases. These results were obtained mainly to determine the phase transition temperature which is useful for comparing the results from the different samples as a function of reduced temperature in the $\mathrm{L}_{\alpha}$ phase.

\section{A. Low Temperature Phases and Phase Transition}

When comparing samples of different composition, it is useful to measure temperature with respect to the critical temperature $\mathrm{T}_{\mathrm{c}}$ which we define as the temperature on the cooling 
curve at which the first sign of a non- $\mathrm{L}_{\alpha}$ phase spectrum appears. Thus we define the reduced temperature $\mathrm{T}_{\mathrm{r}}$ by $T_{r}=T-T_{c}$. We have found it convenient to use two methods of estimating $T_{c}$. The first method utilizes the signal from the $\mathrm{CD}_{2}$ 's in the gel phase and the second method is based on the changes in signal from the $\mathrm{D}_{2} 0$.

The first method is illustrated by Fig. 1 which shows the spectrum from a mixture of the $\mathrm{L}_{\alpha}$ and gel phases for the $5 \% \mathrm{C}_{2 \mathrm{Z}}$ sample at $36.5^{\circ} \mathrm{C}$. Although we call all lower temperature phases gel phases, their actual structure is uncertain. The large intensity peak below $2 \mathrm{kHz}$ (offscale) in Fig. 1 is from the $\mathrm{D}_{2} 0$. This peak obscures the $\mathrm{CD}_{3}$ signal in both Figs. 1 and 2 . The resolved peaks between 5 and $16 \mathrm{kHz}$ are from the $\mathrm{L}_{\mathrm{a}}$ region and correspond to the $90^{\circ}$ powder pattern edges due to the different $\mathrm{CD}_{2}$ 's in the hydrocarbon chain (8). These peaks from the $\mathrm{CD}_{2}$ 's in the $\mathrm{L}_{\alpha}$ phase manifest themselves much more clearly at higher temperatures as shown in Fig. 2 which corresponds to the same sample $\left(5 \%, \mathrm{C}_{22}\right)$ at $145^{\circ} \mathrm{C}$. The broad signal with a cut-off in the vicinity of $30 \mathrm{kHz}$ in Fig. 1 is typical of methylene chains in a gel or $\mathrm{L}_{\beta}$ phase region (8). (This signal does contain a small contribution from the wings of the $\mathrm{L}_{\alpha}$ phase spectrum but this contribution will not affect our analysis seriously.) To obtain information about the percent of gel in the two phase region and also to define the phase transition temperature $T_{c}$, we integrate a region of the broad gel spectrum. The lower limit of integration is chosen so as to minimize the contribution of the $\mathrm{L}_{\alpha}$ phase spectrum wings. Integrals plotted vs. $T$ serve to define both $T_{\mathrm{c}}$ and the composition of the two phase regions.

The second method of studying the phase transition involves observing the $\mathrm{D}_{2} \mathrm{O}$ signal as a function of temperature. $T_{\mathrm{c}}$ is readily obtained from the first appearance of the gel $\mathrm{D}_{2} \mathrm{O}$ signal on cooling. This method is illustrated in Fig. 3 for the $20 \% \mathrm{C}_{22}$ sample. Not only is this a very sensitive way of defining $T_{\mathrm{c}}$, but it also demonstrates that in several cases the gel is a multiphase region, at least as far as the water is concerned. The 0.5 and $1.8 \mathrm{kHz}$ peaks in the two highest temperature spectra in Fig. 3 are the signals from $\mathrm{D}_{2} \mathrm{O}$ and the palmitate $\mathrm{CD}_{3}$ respectively in the $\mathrm{L}_{\alpha}$ phase. The peaks at $0,1.1$, and $1.6 \mathrm{kHz}$ in the lowest temperature spectrum in Fig. 3 are assumed to be signals from three types of $\mathrm{D}_{2} \mathrm{O}$ in the gel phase. On cooling, the first detected gel phase $\mathrm{D}_{2} \mathrm{O}$ peak in the spectra of both $20 \% \mathrm{C}_{22}$ and $20 \% \mathrm{C}_{8}$ samples appears to have zero splitting, which indicates that there is $\mathrm{D}_{2} \mathrm{O}$ in an isotropic environment such as patches of bulk water. Whereas there appear to be at least three $\mathrm{D}_{2} \mathrm{O}$ gel phase environments for the $20 \% \mathrm{C}_{22}$ sample (Fig. 3), and at least two for the $20 \% \mathrm{C}_{8}$ sample, there is only one gel phase $\mathrm{D}_{2} \mathrm{O}$ environment for the $0 \%$ sample. For the latter sample, an isotropic $\mathrm{D}_{2} \mathrm{O}$ peak was not observed. (We note that for another $0 \%$ sample, an isotropic phase $\mathrm{D}_{2} \mathrm{O}$ peak was observed at $30^{\circ} \mathrm{C}(9)$.) As demonstrated in Fig. 3, the growth and decay of the various $\mathrm{D}_{2} \mathrm{O}$ phases on cooling is easily followed by deuterium nmr. For. potassium palmitate in the presence of guest chains, this illustrates that for the water at least, the "gel" phase is a complicated many-phase region. Another interesting feature of the "mixed phase" region will be discussed in the following section after presentation of Figs. 5 and 6.

$T_{c}$ for each sample has been estimated by the two methods outlined here and the values are given in Table 1. We note that recently another powerful way of measuring phase transition temperatures using the method of moments has been reported (11).

\section{B. La Phase}

The deuterium nmr of the $\mathrm{D}_{2} \mathrm{O}$ and the potassium palmitate host in the six samples has been used to obtain the spectral splittings, $\Delta v$, between the two $90^{\circ}$ powder pattern singularities of the $\mathrm{D}_{2} \mathrm{O}$, the $\mathrm{CD}_{3}$ and each $\mathrm{CD}_{2}$ deuterium as a function of temperature in the $\mathrm{L}_{\alpha}$ phase. A 
sample spectrum, with the spectral peaks identified by their position along the chain, is shown in Fig. 2, We have assumed that all our spectra are indeed from samples in a true lamellar phase. The opposite direction and moderate size of the changes observed with different chain length guests support this assumption. There are certainly no changes to a cubic or hexagonal phase (1): these would be associated with a drastic reduction in spectral splittings (12). We shall examine several ways of comparing results from the different samples and of handling the more than 1000 measured splittings. Starting from the spectral splitting as a function of carbon position, we discuss results for the different samples for two values of $T_{\mathrm{r}}$, one near the phase transition and the other at a higher temperature. As is the case throughout this study, results from the $1 \% \mathrm{C}_{22}$ and $1 \% \mathrm{C}_{8}$ samples did not differ, within experimental error, from those for the $0 \%$ sample and for clarity we omit those points from most graphs. Also, a general result is that the splittings for the $5 \% \mathrm{C}_{22}$ sample were always between those for the $0 \%$ and $20 \% \mathrm{C}_{22}$ samples, as might be expected for a dilution effect.

However, the differences were not always linear and we include the data from the $5 \% \mathrm{C}_{22}$ sample in most plots.

Figure 4IaI shows the spectral splittings $\Delta v$ at two reduced temperatures as a function of carbon positions in the host perdeuterated potassium palmitate for the $0 \%, 5 \% \mathrm{C}_{22}, 20 \% \mathrm{C}_{22}$, and $20 \% \mathrm{C}_{8}$ samples. In order to see some of the effects on the host of the long and short chain guests more easily, Fig. $4 b$ shows the ratios of the splittings in the $5 \%$ and two $20 \%$ samples to the splittings in the $0 \%$ sample. The lines joining data points in Fig. 4 are for visual clarity only. At low temperatures, the addition of either long or short chain farry acid salt guests increases spectral splittings in the host (for all except the $\alpha$ position of $20 \% \mathrm{C}_{8}$ where there is a slight decrease). At higher temperatures, the addition of long chain guests gives rise to an increase in spectral splitting (beyond the carbon 4 position) whereas the addition of short chain guests gives rise to a decrease in spectral splittings. The high temperature result for carbons 5 to 15 might be expected intuitively on the basis of chain packing, as one might expect the 16-carbon chain to have more freedom to move in the presence of short chains and less freedom in the presence of long chains. The low temperature results and the high temperature polar region results are probably associated with differing interactions between lipid and water for the different samples. This is discussed more fully in the following paragraph.

Plots such as those in Fig. 4 allow a comparison of chain order from one position to the next at specific temperatures. Another informative way of presenting the data is to choose various $\mathrm{CD}_{2}$ positions and to examine the splittings as a function of reduced temperature. This is done in Fig. 5 for the carbon 2, carbons 5 and 6, carbon 10, and carbon 15 positions. The $\mathrm{D}_{2} \mathrm{O}$ splittings are much smaller than the $\mathrm{CD}_{2}$ splittingsand are shown in Fig. 6. In Figs. 5 and 6, part $a$ shows the splittings of the $0 \%, 5 \% \mathrm{C}_{22}, 20 \% \mathrm{C}_{22}$, and $20 \% \mathrm{C}_{8}$ samples and part $b$ shows the ratio of the splittings of the latter three to those of the $0 \%$ sample. At high temperatures and toward the methyl end of the chain the results are as expected from the previous arguments about chain packing. The results for the 2 position (Fig. 5) and for the $\mathrm{D}_{2} \mathrm{O}$ (Fig. 6) are quite striking, however, in that the $0 \%$ sample always has the greatest splitting at high temperature. As has been discussed elsewhere (9), the reduction of spectral splittings are not necessarily associated with greater mobility of the system, but may merely reflect changes in the preferred angle that a part of the molecule makes with the normal to the plane of the bilayer. Our results, then, imply: $(a)$ that the palmitate has greater mobility at the head group in the presence of guest chains, as might be expected if the planar arrangement of the carbonyl groups is distorted because of the heterogeneity of chain lengths; and (or) $(b)$ that the addition of guests alters the distribution of 
orientations that the first $\mathrm{C}-\mathrm{C}$ bonds and the $\mathrm{D}_{2} \mathrm{O}$ make with the normal to the bilayer. Additionally, for both the $0 \%$ and $5 \% \mathrm{C}_{22}$ samples, the splittings of the carbon 2 position and the $\mathrm{D}_{2} \mathrm{O}$ first rise with increasing temperature and then fall; this observation has been attributed to changes in the interaction between lipid and water (9). However, for the $20 \%$ samples the 2 position splittings decrease with increasing temperature, but the water shows an increase and then a decrease. In the spirit of the model of of ref. 9, these results indicate a different interaction between the lipid and the water in the $20 \%$ samples than in the $0 \%$ sample. Qualitatively, the following picture can explain these results. The presence of mixed chain lengths with altered packing requirements inside the bilayer may well disturb the planar arrangement of the carboxyl groups. The orientation of water molecules which are hydrogen bonded to two carboxyl groups will then change, leading to an orientation that gives a lower splitting. The orientation of the water molecules may well change with temperature leading to the observed rise and fall of the $\mathrm{D}_{2} \mathrm{O}$ splittings relative to the lamellar normal. However, this change in orientation of the $\mathrm{D}_{2} \mathrm{O}$ is not necessarily associated with similar changes in the orientation of the head groups. Such a situation would explain the observed splittings. It should be pointed out that the above model is somewhat crude, and that at the moment we have not studied the deuterium spectral splittings of the guest chains.

The water splittings are an average over many water environments and it is difficult to make more than qualitative comparisons between lipid and water spectral parameters.

It is convenient, at this stage, to point out an interesting feature of the "mixed phase" region $\left(T_{\mathrm{r}}<0\right)$ for the $20 \% \mathrm{C}_{22}$ sample (solid triangles in Figs. $5 a$ and $6 a$ ). Except for the 2 position, the $20 \% C_{22}$ splittings for the $\mathrm{L}_{\alpha}$ component have a maximum at $T=T_{c}\left(T_{\mathrm{r}}=0\right)$ in Fig. 5a. That the splittings decrease on decreasing temperature below $T_{c}$ is presumably due to the $\mathrm{L}_{\mathrm{a}}$ component of the mixed phase region becoming richer in the host palmitate as the sample is cooled. The splittings are approaching the lower values observed for the $0 \%$ sample. The behavior of the $\mathrm{D}_{2} \mathrm{O}$ signal (Fig. 6a) from the $L_{\alpha}$ phase region below $T_{c}$ is also in agreement with this interpretation.

Examination of Figs. 4-6 is fruitful but these figures present only part of the results and focus on only one aspect of the lipid ordering at a time. We shall next examine convenient ways to compare all $\mathrm{CD}_{2}$ splittings on a single plot. To do so, we define the average splitting $\langle\Delta v\rangle$, the mean square splitting $\left\langle(\Delta \mathrm{v})^{2}\right\rangle$ and the distribution parameter $\Delta_{2}$ by

$$
\begin{aligned}
& \text { [1] }\langle\Delta v\rangle=\frac{1}{14} \sum_{i=2}^{15} \Delta v_{i} \\
& \text { [2] }\left\langle(\Delta v)^{2}\right\rangle=\frac{1}{14} \sum_{i=2}^{15}\left(\Delta v_{i}\right)^{2} \\
& \text { [3] } \Delta_{2}=\frac{\left\langle(\Delta v)^{2}\right\rangle}{\langle\Delta v\rangle^{2}}-1
\end{aligned}
$$

The average splitting $\langle\Delta v\rangle$ and the distribution parameter $\Delta_{2}$ are plotted as function of reduced temperature in Figs, 7 and 8 respectively. The plots in Fig. 7 for $\langle\Delta v\rangle$ are very similar to those for positions 10 and 15 in Fig. $5 a$ and the previous arguments about lipid packing and the lipidwater interaction can again be used to interpret the results. The plot in Fig. 8 for $\Delta_{2}$ is a compact and particularly useful way to present the results. (The distribution parameter $\Delta_{2}$ has Deen discussed in detail elsewhere (11).) For example, a value of $\Delta_{2}=0$ means that all splittings are equal and the greater the value of $\Delta_{2}$ the greater is the distribution in the splittings along the chain. In fact much information on the average order parameter profile is contained in Fig. 8. It is immediately obvious that the spectral peaks spread out at higher temperatures; or, as observed 
previously (8), the "plateau" in the splittings versus position curve disappears. Also, the $20 \% \mathrm{C}_{8}$ and $0 \%$ samples are seen to have a similar distribution of splittings at all temperatures, while the $20 \% \mathrm{C}_{22}$ sample has less spread in peak positions at all values of $T_{\mathrm{r}}$. These observations can also be made by close inspection of the many curves given for a limited temperature range in Fig. $4 a$ but the comparisons are more convenient with the condensation of the entire set of results represented by Figs. 7 and 8. However, Figs. 4-6 are most useful in examining specific areas of interest; for example the averaging involved in Figs. 7 and 8 tend to hide some of the interesting features of the polar head region.

Another way of examining the results is to look at ratios of various splittings. Such ratios do not give any information about the magnitude of the orientational order of the chain, but they show instead the relative order of different parts of the chain, and how that relative order changes upon addition of guest chains. To demonstrate this, we plot in Fig. 9 the ratios of splittings for positions 10 to 15 versus $T_{\Gamma}$ for the various samples. The ratio of couplings, $\Delta v_{i 0} / \Delta v_{15}$, clearly demonstrates that, relative to the middle of the chain, the methyl end is less ordered in the presence of short chains and more ordered in the presence of long chains at all temperatures. This result is reasonable since long chain guests would restrict the motions of the host methyl end while with the short chain guest, the host methyl end has more room to move about than it does in the $0 \%$ sample.

Ratios of nmr splittings of one part of a chain to those of another part have recently been used in an interesting way (13). The basis of the treatment is to describe the order parameter for a $\mathrm{CD}$ bond as the product of molecular and conformational order parameters $(13,14)$. In practice, this separation cannot be performed quantitatively because there is no way to measure one of the order parameters separately. Also, the concept of a unique molecular order parameter matrix in soap and liquid crystal systems may not be valid (15) (and private communication by E.

Samulski) because it implies that one can do time averages over the molecular and conformational motions independently (15). However, it is useful to employ this conformational motions independently (15). However, it is useful to employ this method of analysis since it provides an empirical way of treating the results, at least for some chain positions. For a uniaxial phase, the model employs two order parameters, and it suggests that the splitting $\Delta v_{i}$ for the $i$ th $\mathrm{CD}_{2}$ can be related to the splittings of any other two $\mathrm{CD}_{2}$ splittings $\Delta v_{j}$, and $\Delta v_{k}$ by the equation (13)

$$
\text { [4] } \Delta v_{i}=C_{j k}^{i} \Delta v_{j}+C_{k j}^{i} \Delta v_{k}
$$

where the $C_{j k}{ }^{i}$ are functions of the conformational order parameters in this model. If the $C_{j k}{ }^{i}$ are independent of temperature, plots of $\Delta v_{j} / \Delta v_{k}$ versus $\Delta v_{j} / \Delta v$ will give straight lines with slope $C_{j k}{ }^{i}$ and intercept $C_{j k}{ }^{i}$ as observed in ref. 13. In such a situation one can use [4] as an empirical equation in spite of the objections raised above. We have applied this method to the case for $j=$ 10 and $k=15$ and plotted the equation

$$
\text { [5] } \frac{\Delta v_{1}}{\Delta v_{15}}=C_{10,15^{i}} \frac{\Delta v_{10}}{\Delta v_{15}}+C_{15.10^{i}}
$$

for all chain positions for each sample. The experimental points can be approximated with straight lines and least-squares fits to [5] give values of $C_{10,15^{i}}$ and $C_{15,10^{i}}$. The largest deviation from linearity is obtained for the $20 \% \mathrm{C}_{8}$ sample, where there are slight breaks and changes in curvature in the plots. These effects could be due to phase transitions reported in ref. 10 .

Although this limits the universality of the approach, these changes are not sufficient to affect the general use of the empirical procedure. The case of $i=\mathrm{D}_{2} \mathrm{O}$ was also considered since $\mathrm{D}_{2} \mathrm{O}$ is 
expected to be hydrogen- bonded to the polar head group. This case does not give linear fits, indicating that the $\mathrm{C}_{10,15} \mathrm{D}_{2} \mathrm{O}$ and $\mathrm{C}_{15,10} \mathrm{D}_{2} \mathrm{O}$ do depend on temperature. This is not surprising since specific interactions between $\mathrm{D}_{2} \mathrm{O}$ and the polar head group are strongly temperature dependent, as discussed previously.

This method of analysis is useful for several reasons. It can serve to identify inconsistent assignments of peaks which would show up as deviations from linearity. This is especially helpful in regions of the deuterium nmr spectra where many splittings are almost equal. Also, the $C_{j k}{ }^{i}$ values can be used to condense spectral information. The 13 pairs of numbers $C_{10,15^{i}}$ and $C_{15,10}{ }^{i}$, in conjunction with the $i=10$ and $i=15$ parts of Fig. $5 a$, can serve to reconstruct, at least qualitatively, all the spectral results for the soap chains.

The most interesting result of this analysis is that the values of $C_{10,15^{i}}$ and $C_{15,10^{i}}$ change by up to $50 \%$ from sample to sample in a consistent way. This change with sample is demonstrated in Fig. 10 for $i=2,7$, and 12. We note that the $1 \% \mathrm{C}_{8}$ and $1 \% \mathrm{C}_{22}$ samples are represented in this plot whereas they have not appeared previously. Four of the six plots in Fig. 10 are roughly linear, and, what is even more interesting the slopes are of equal and opposite sign for short and long chains as indicated by the dashed lines. Hence, this analysis is useful in comparing the order of the palmitate chain in the presence of other soap chains in a manner that is temperature independent. In terms of the model used (13), the changes in $C_{i k^{i}}$ would indicate systematic differences in the conformational order parameters of the palmitate chains in the various mixtures. From the empirical viewpoint, the changes serve to characterize the sensitivity of the potassium palmitate $\mathrm{L}_{\alpha}$ phase to disturbances by added short and long chain molecules.

\section{Conclusion}

We have presented deuterium nmr results for perdeuterated potassium palmitate $\mathrm{D}_{2} \mathrm{O}$ mixtures in the presence of varying amounts of potassium octanoate and potassium behenate. The presence of the guest molecule alters the order of the hydrocarbon chain of the host molecule. At higher temperature, the short chain guest leads to a reduced order for the host, while the long chain guest has the opposite effect for all except the head of the host. At low temperature the interaction between lipid and water would seem to be very important in determining the overall host chain spectral parameters. This interaction appears to change the average angle that the first $\mathrm{C}-\mathrm{C}$ bond makes with the normal to the plane of the bilayer, and hence affects the splittings for all chain positions. The presence of soaps of differing chain lengths would seem to cause changes in the lipid-water interactions, possibly because the presence of these chains distorts the arrangement of the polar heads with respect to the bilayer plane. The details of the interactions involved in the polar head region should be further studied using an alternative approach such as with proton spin labelling techniques (16).

At lower temperatures there exists a multiphase gel region for the samples containing mixtures of fatty acids. Although the hydrocarbon chain deuteriums give the same spectrum in this temperature range for all samples, the $\mathrm{D}_{2} \mathrm{O}$ signal is a useful probe for this region, as separate resonances are observed for the $\mathrm{D}_{2} \mathrm{O}$ in different gel regions. From the $\mathrm{CD}_{2}$ signal, quantitative information about the mixed phase region is obtainable.

The most instructive extension of this work would be to study the guest molecules in the way the host molecules have been investigated here. 
Acknowledgements: We wish to thank Myer Bloom for helpful discussions and Jim Davis and Alex Mac Kay for technical assistance. Research support by the National Research Council of Canada is gratefully acknowledged.

\section{References}

1. P. EKWALL, Adv. Liq. Cryst. 1, 1 (1975).

2. J. CHARVOLJN and B. MELY. Mol. Cryst. Liq. Cryst. Lett. 41,209(1978).

3. J. H. DAVIS, K. R. JEFFREY, M. BLOOM, M. L VALIC, and T. P. HIGGS, Chem. Phys. Lett. 42,390(1976).

4. J. CHARVOLIN, P. MANNEVILLE, and B. DELOCHE. Chem. Phys. Lett. 23, 345 (1973).

5. B. ME'LY and J. CHARVOLIN. Colloque C.N.R.S., June 1978. In press.

6. D. M. CHEN, F. Y. FUJIWARA, and L. W. REEVES. Can. J. Chem. 55,2404 (1977).

7. C. Y. Y. HSIAO, C. A. OTTAWAY, and D. B. WETLAUFFER. Lipids, 9, 913 (1974).

8. J. H. DAVIS and K. R. JEFFREY. Chem. Phys. Lipids, 20,87 (1977).

9. K. BDOLALL, E, E, BURNELL, and M, Í. VALIC. Chem. Phys. Lipids, 20, 115(1977), 10. N. A. P. VAZ, J. W. DOANE, and M. E. NEUBERT. Phys. Rev. Lett. 42, 1406(1979). 11. M. BLOOM, J. H. DAVIS, and F. W. DAHLQUIST. To be published; I. H. DAVIS. Biophys. J. 27,339(1979).

12. B. MÉLY and J. CHARVOLIN. Chem. Phys. Lipids, 19, 43 (1977).

13. P. J. Bos and J. W. DOANE. Phys. Rev. Lett. 40,1030(1978).

14. B. DELOCHE and J. CHARVOLIN. J. Phys. Lett. 41, L39 (1980).

15. E. E. BURNELL and C. A. DE LANQE. J. Magn. Reson. 39, 461 (1980).

16. T. P. HIGGS and A. L. MACKAY. Chem. Phys. Lipids, 20, 105(1977). 


\section{Figures}

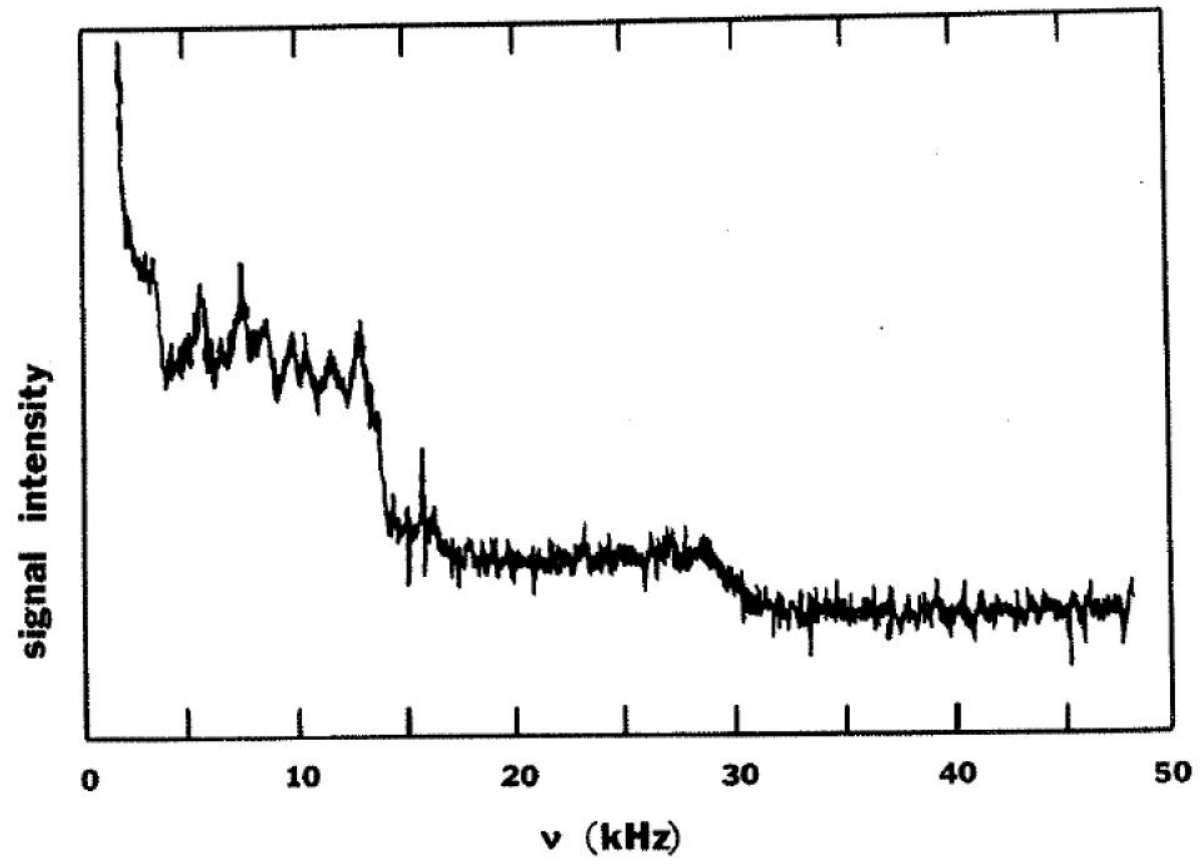

Fig. 1. The deuterium nmr spectrum of the mixture of lamellar and gel phases of the $5 \% \mathrm{C}_{22}$ sample (see Table 1) at $36.5^{\circ} \mathrm{C}$. Only one half of the symmetric spectrum is shown with spectrum center at $0 \mathrm{kHz}$.

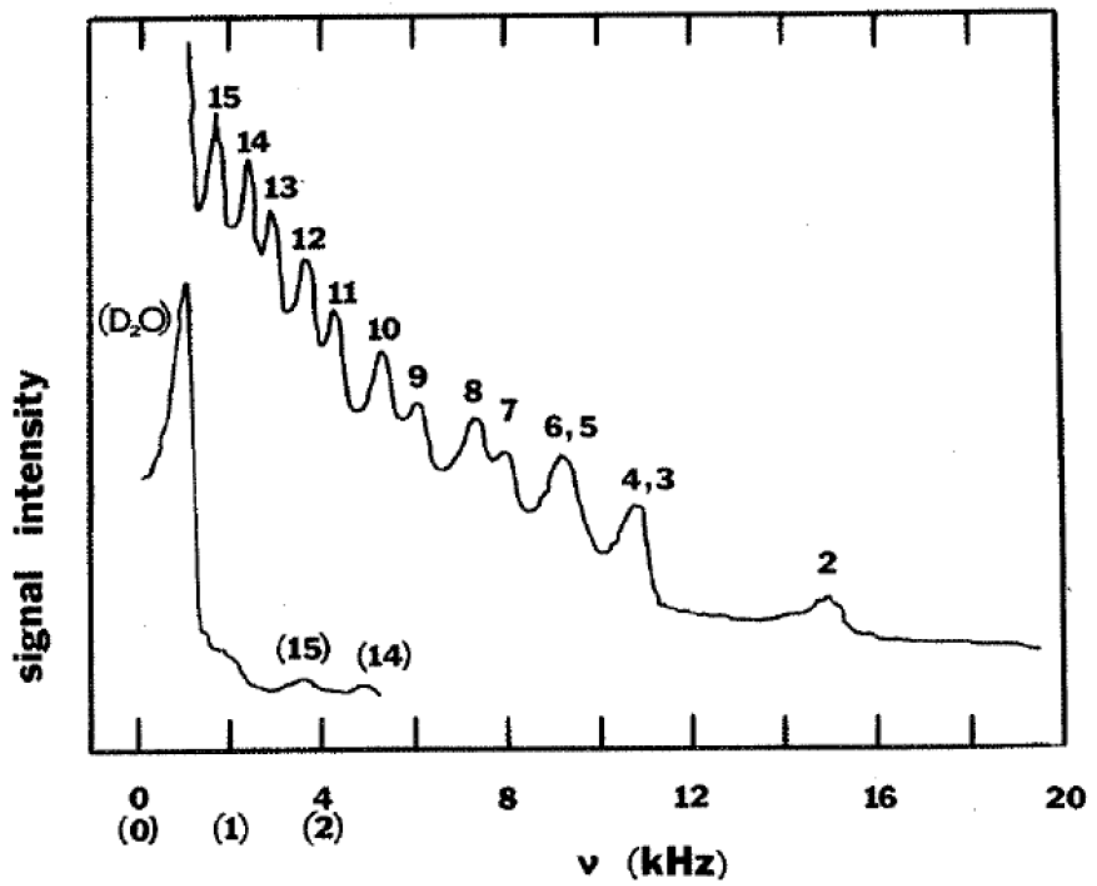

Fig. 2. The deuterium nmr half-spectrum of the $5 \% \mathrm{C}_{22}$ sample at $145^{\circ} \mathrm{C}$. The peaks are labelled by carbon position along the chain. The $\mathrm{D}_{2} \mathrm{O}, 15$, and 14 position peaks shown in parentheses at the lower left correspond to the expanded scale shown in parentheses on the abscissa. 


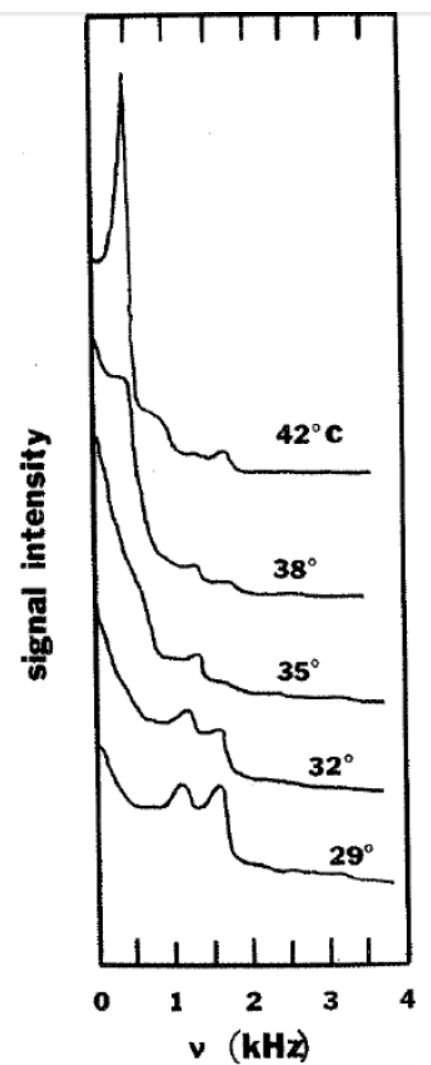

FIG. 3. The central part (low frequency) of the deuterium nmr half-spectra showing the $D_{2} O$ signals from the $20 \% C_{22}$ sample at various temperatures below $T_{\mathrm{c}}$.
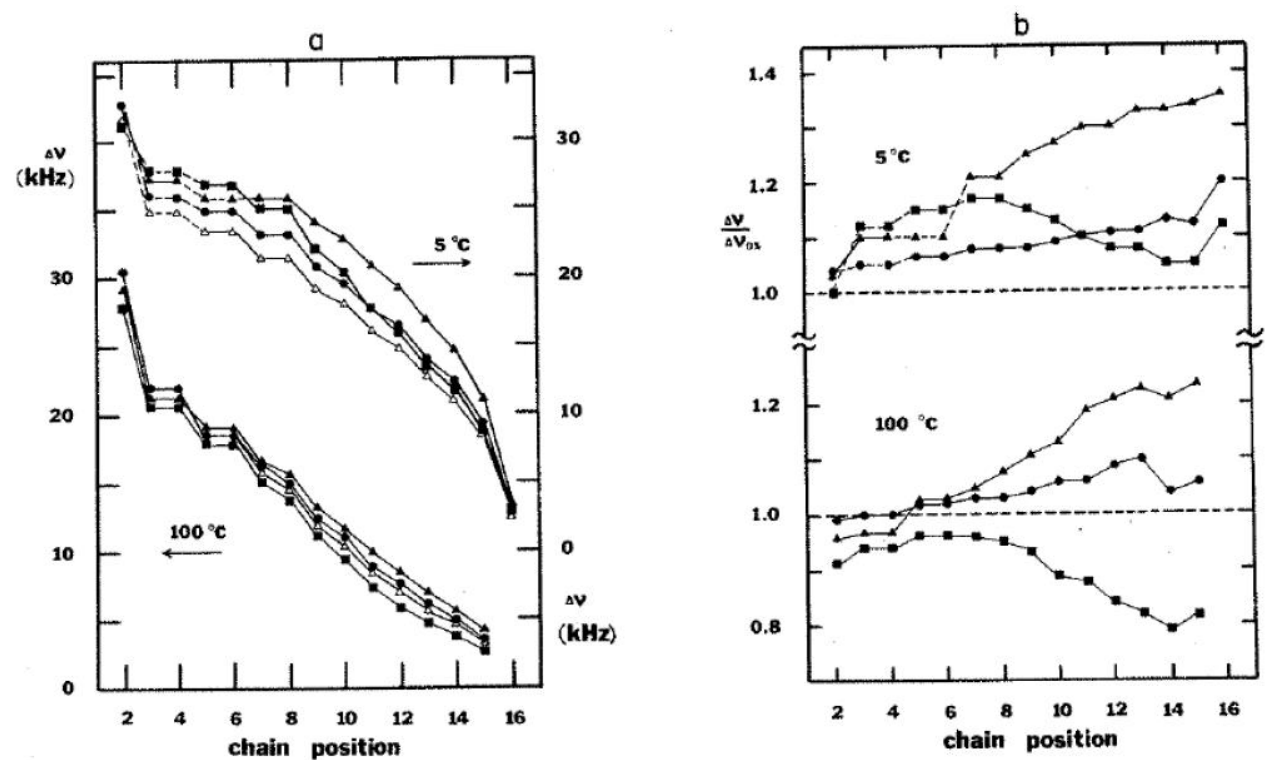

F1G. 4. Perdeuterated potassium palmitate deuterium $\mathrm{nmr}$ splittings and splitting ratios as a function of carbon position at . the reduced temperatures of 5 and $100^{\circ} \mathrm{C}$. The splittings are shown in $a$ where the right-hand ordinate scale cor the $0 \%$ sample are $T_{\mathrm{r}}=5^{\circ} \mathrm{C}$ and the left-hand ordinate scale corresponds to $T_{\mathrm{r}}=100^{\circ} \mathrm{C}$. The ratios of the splittings to those of the $0 \%$ sample are

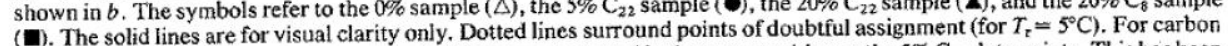
position solin the $5 \% \mathrm{C}_{22}$ data points. This has been positions for clarity and the small difference in these values can be seen by investigating $b$. 

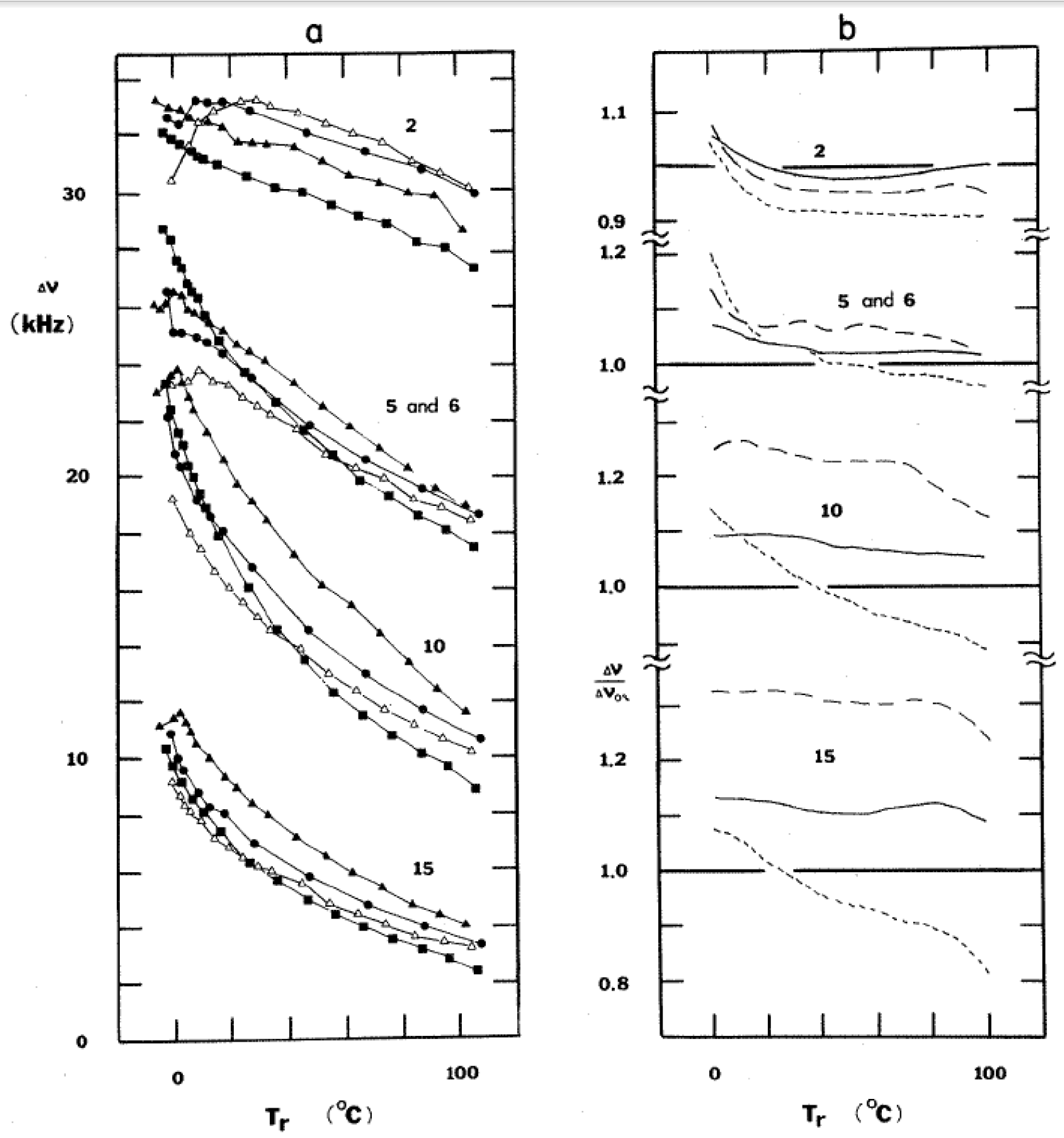

Fro. 5. Perdeuterated potassium palmitate deuterium nmr splittings $\Delta v$ and ratios $\Delta v / \Delta v_{0}$ as a function of reduced temperature $T_{\mathrm{r}}$ for various carbon positions as indicated. The absolute splittings $\Delta v$ are shown in $a$ for the $0 \%$ sample $(\Delta)$, the $5 \% \mathrm{C}_{22}$ sample $(\boldsymbol{\Theta})$, the $20 \% \mathrm{C}_{22}$ sample $(\boldsymbol{\Delta})$, and the $20 \% \mathrm{C}_{8}$ sample $(\boldsymbol{E})$. The ratios of the splittings to the splittings in the $0 \%$ sample $\Delta \mathrm{v} / \Delta \mathrm{v}_{0 \%}$ are shown in $b$ for the $5 \% \mathrm{C}_{22}$ sample $\left(-{ }_{-}\right.$, the $20 \% \mathrm{C}_{22}$ sample $(--)$, and the $20 \% \mathrm{C}_{8}$ sample $(--)$. 

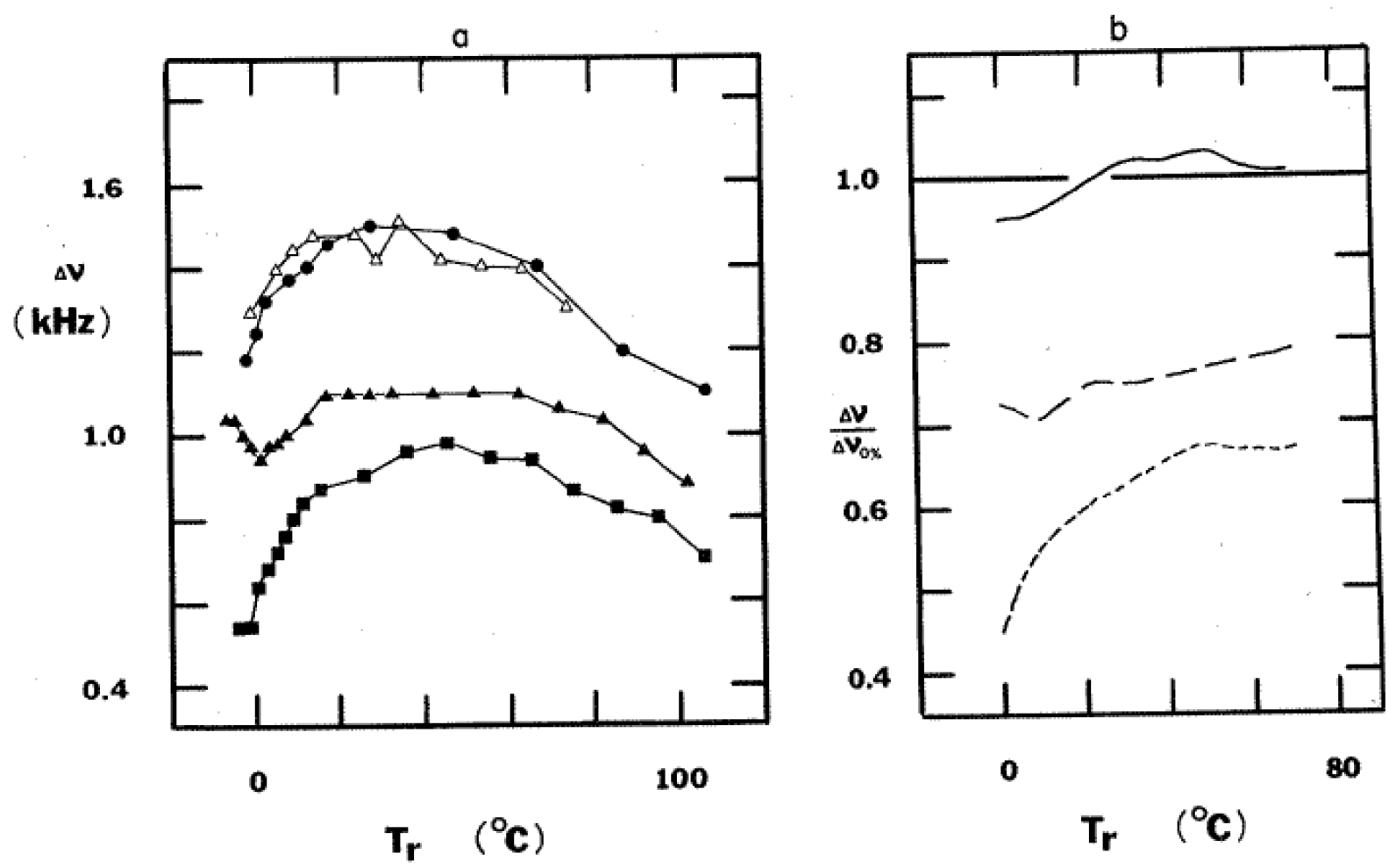

Fig. 6. $\mathrm{D}_{2} \mathrm{O}$ deuterium nmr splittings $\Delta v$ and ratios $\Delta v / \Delta v_{0 \%}$ as a function of reduced temperature $T_{\mathrm{r}}$. The absolute splittings $\Delta v$ are shown in $a$ for the $0 \%$ sample $(\Delta)$, the $5 \% \mathrm{C}_{22}$ sample $(\bullet)$, the $20 \% \mathrm{C}_{22}$ sample $(\boldsymbol{\Delta})$, and the $20 \% \mathrm{C}_{8}$ sample ( $\left.\boldsymbol{\square}\right)$. The ratios of the splittings to the splittings in the $0 \%$ sample $\Delta v / \Delta v_{0 \%}$ are shown in $b$ for the $5 \% \mathrm{C}_{22}$ sample $(-)$, the $20 \% \mathrm{C}_{22}$ sample (- - ) , and the $20 \% \mathrm{C}_{\mathrm{s}}$ sample ( $\cdots$ ).

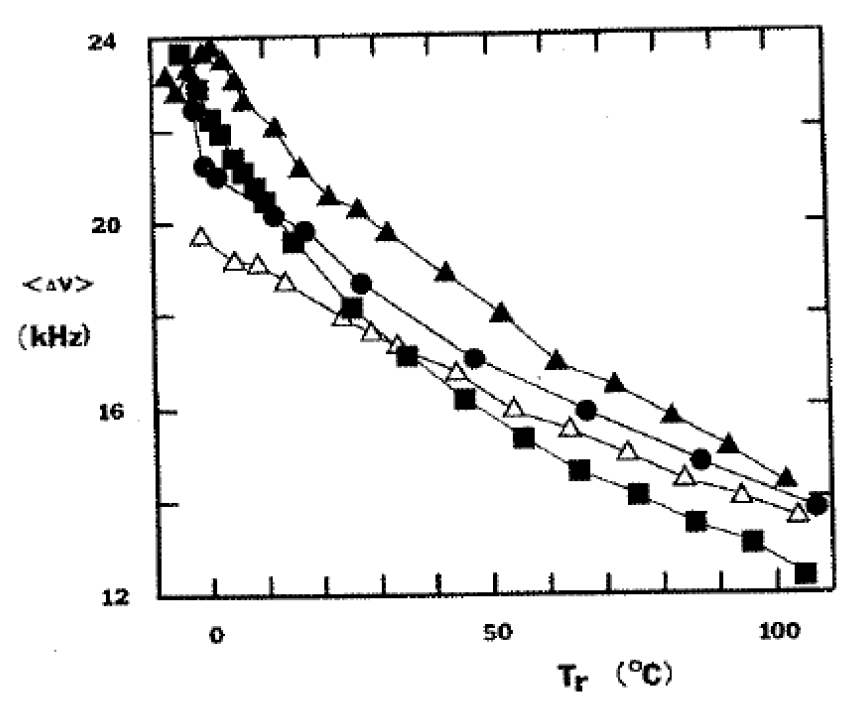

FIG. 7. The average value $\langle\Delta v\rangle$ of the perdeuterated potassium palmitate splittings $\Delta v_{i}$ given by [1] in the text as a function of reduced temperature $T_{r}$ for the $0 \%$ sample $(\Delta)$, the $5 \% \mathrm{C}_{22}$ sample (C), the $20 \% \mathrm{C}_{22}$ sample $(\boldsymbol{A})$, the $20 \% \mathrm{C}_{8}$ sample (W). 


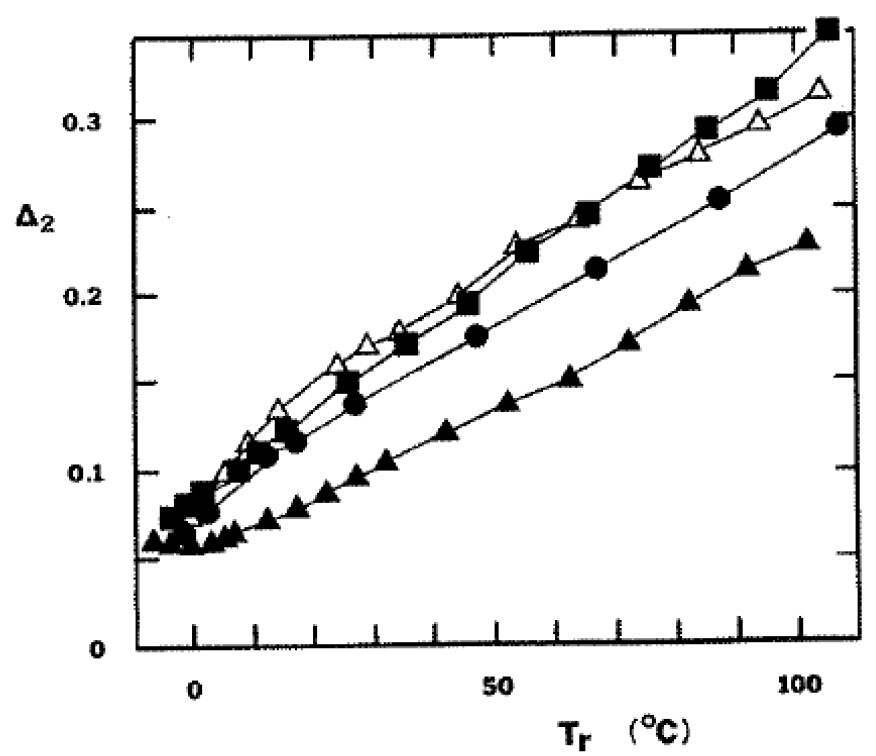

F1G. 8. The distribution parameter $\Delta_{2}$ of the perdeuterated potassium palmitate given by [3] in the text as a function of reduced temperature $T_{\mathrm{r}}$ for the $0 \%$ sample $(\Delta)$, the $5 \% \mathrm{C}_{22}$

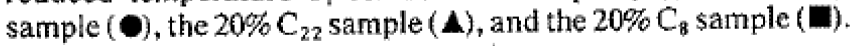

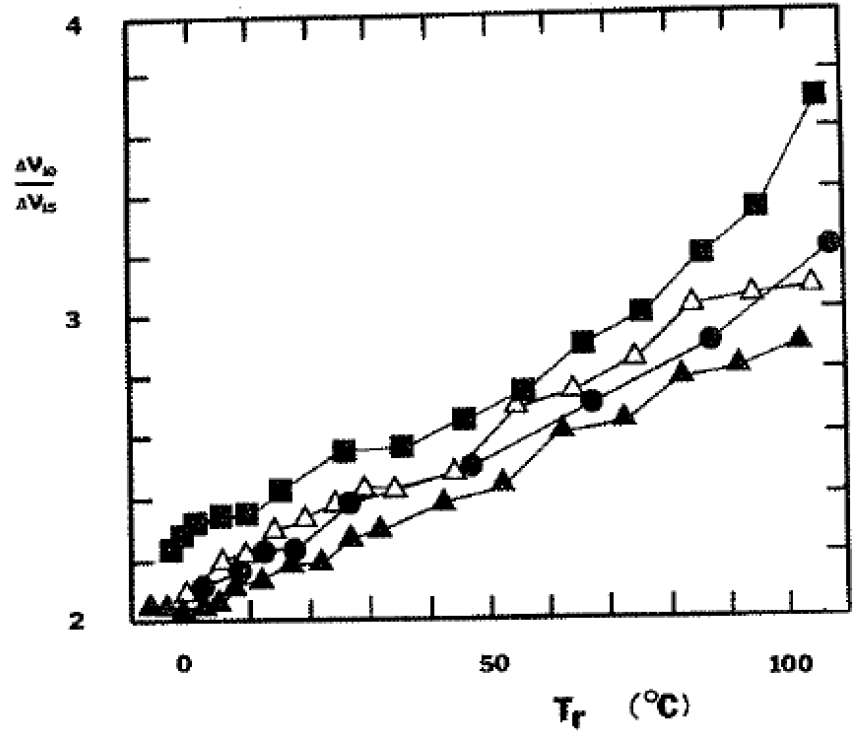

FIG. 9. The ratio of the perdeuterated potassium palmitate splittings for carbon position 10 to that of carbon position 15 as a function of reduced temperature $T_{*}$ for the $0 \%$ sample $(\Delta)$, the $5 \% \mathrm{C}_{22}$ sample (C), the $20 \% \mathrm{C}_{22}$ sample (A), and the $20 \% \mathrm{C}_{5}$ sample (1). 


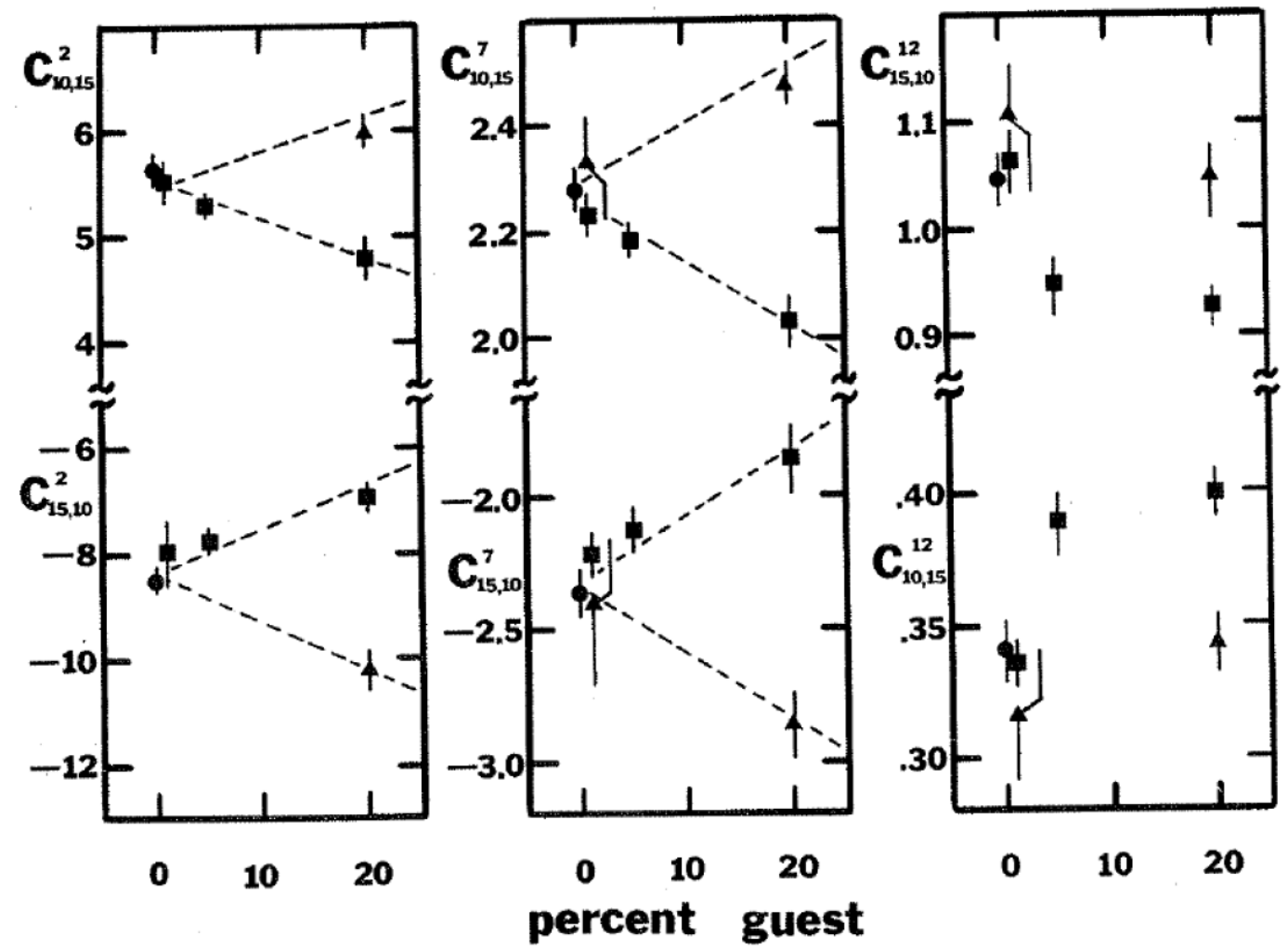

Fic, 10. Value of $C_{10,1}{ }^{i}$ and $C_{15,10^{i}}$ for $i=2,7$, and 12 as a function of percent guest molecule. The symbols refer to $0 \%(\bullet)$, $\% \mathrm{C}_{22}(\mathbf{m})$, and $\% \mathrm{C}_{8}(\mathbf{A})$. The pairs of dashed lines in $C^{2}$ and $C^{7}$ have equal and opposite slopes. 


\section{Tables}

TABLE 1. Composition and phase transition temperature of samples

\begin{tabular}{cccccc}
\hline \hline Sample & $\begin{array}{c}\text { Mol\% } \\
\mathrm{D}_{2} \mathrm{O}\end{array}$ & $\begin{array}{c}\text { Mol\% } \\
\text { K-octanoate }\end{array}$ & $\begin{array}{c}\text { Mol\% } \\
\text { perdeuterated } \\
\text { K-palmitate }\end{array}$ & $\begin{array}{c}\text { Mol\% } \\
\text { K-behenate }\end{array}$ & $T_{\mathrm{c}}\left({ }^{\circ} \mathrm{C}\right)$ \\
\hline $0 \%$ & 86.3 & - & 13.7 & - & 41 \\
$1 \% \mathrm{C}_{8}$ & 86.3 & 0.14 & 13.6 & - & 41 \\
$20 \% \mathrm{C}_{8}$ & 86.3 & 2.7 & 11.0 & - & 40 \\
$1 \% \mathrm{C}_{22}$ & 86.3 & - & 13.6 & 0.14 & 41 \\
$5 \% \mathrm{C}_{22}$ & 86.3 & - & 13.0 & 0.68 & 38 \\
$20 \% \mathrm{C}_{22}$ & 86.3 & - & 11.0 & 2.7 & 43 \\
\hline
\end{tabular}

